\title{
“CONTRA LA ORGANIZACIÓN”. CAOS Y CREACIÓN EN PAUL KLEE Y GILLES DELEUZE
}

\author{
"Against the organization". Chaos and creation in Paul Klee and Gilles Deleuze \\ Mauricio Carreño Hernández \\ Universidad de Santiago de Chile, Santiago, Chile \\ mauricio.carreno@usach.cl \\ Tomás Flores Estay \\ Universidad de Chile, Santiago, Chile \\ tpflores@uc.cl
}

\section{Resumen}

El presente artículo busca tensionar la noción de organización a partir de la producción teórica de Paul Klee y su diálogo con la obra de Gilles Deleuze. El encuentro entre ambos autores nos permitirá problematizar el concepto como un dispositivo de fijación y reducción de lo viviente a una cualidad invariable e inamovible a nivel de la configuración de las formas vivas y de la creación artística. A partir del entendimiento del acto de creación como una operación generativa de configuraciones vivas, relevaremos el estatuto de caosmos de la creación, es decir, como punto de génesis de un orden a partir del caos. Así, en vista del rechazo de ambos autores de las esencias en provecho de la variación, la diferencia y el devenir, postularemos la idea de que 'toda configuración es una deformación', tanto en el campo de la génesis vital como de la creación artística.

Palabras clave: organización, caos, creación, Klee, Deleuze

\begin{abstract}
This article intends to put a strain on the notion of organization, based on the relations we can establish between the theoretic production of Paul Klee and the work of Gilles Deleuze. The encounter between those two authors will allow us to pose a problem in regard to the concept, understood as a device which fixates and reduces the living being to an unchanging and static quality at the level of the configuration of living forms and of the artistic creation. From the understanding of the act of creation as an operation generative of living configurations, we will show the relevance of the chaosmotic trait of creation, that is, creation as a genetic point of order from chaos. Thus, considering the rejection form the part of the two authors of the essences, replacing them with the variation, difference and becoming, we will propose the idea that 'every configuration it's a deformation', this as well as in the field of vital genesis as in that of artistic creation.
\end{abstract}

Keywords: organization, chaos, creation, Klee, Deleuze

Fecha de Recepción: 01/07/2019 - Fecha de Aceptación: 26/11/2019 


\section{Introducción}

En la breve nota titulada "Un pintor mental", Antonin Artaud habla de Paul Klee aludiendo en diversas oportunidades a las nociones de organización y síntesis: "síntesis mentales concebidas como arquitectura", "síntesis cósmicas"; "organización de visiones, de formas"; "organización de imágenes" (23). Mediante estas indicaciones, Artaud parece relevar la singularidad presente en las configuraciones pictóricas del artista suizo, sin embargo, ¿a qué clase de organización y síntesis alude Artaud en su pletórico comentario acerca del pintor? ¿No es acaso Klee el artista por excelencia del caos y de la variedad infinita? ¿Cómo llega entonces Artaud a ver en su obra una especie de principio de organización? Lo cierto es que es posible estar más o menos seguras/os que las nociones de organización y síntesis a partir de las cuales Artaud describe la obra de Klee, en modo alguno están al servicio de una tradición -aquella del pensamiento metafísico occidental- centrada en la primacía de la unidad, la inteligibilidad y la identidad.

No obstante, por más confianza que alberguemos en la intuición de Artaud, por fuerza su apelación a las nociones de síntesis y organización para describir el trabajo de Klee continúa siendo una cuestión opaca y oscura. Entonces, es a partir de los aportes del filósofo Gilles Deleuze y, por cierto, de su lectura del trabajo de Klee, que el propósito de Artaud se vuelve más claro. Ello, pues, si hay algo que caracteriza al pensamiento deleuzeano, es justamente avanzar hacia la presentación de un mundo que rebasa 'nuestra' escala habitual, abriendo paso hacia un pensamiento y una práctica de la multiplicidad (Sauvagnargues 11).

Bajo tales condiciones, el presente artículo pretende tensionar el concepto de organización y sus implicancias dentro del campo del acto de creación pictórica a partir de la producción teórica de Paul Klee y su diálogo con la obra de Gilles Deleuze. Se trata de interrogar el modo en el cual ambos autores se han confrontado al problema de la hegemonía y la legitimidad de la organización, relevando su estatuto problemático como una especie de dispositivo de fijación y reducción de lo viviente a una cualidad invariable e inamovible. A partir de su apuesta común en torno al rechazo de las esencias en provecho de la variación, la diferencia y el devenir como cuestiones decisivas para el arte y la filosofía, resultará posible repensar la siempre inestable variabilidad del acto de creación entre el caos y el orden. Es decir, la producción de configuraciones vivas, incluso, más allá del campo propiamente pictórico. Así, en virtud de la idea de que toda configuración [formación] ${ }^{1}$ es una deformación, el presente escrito buscará

\footnotetext{
${ }^{1}$ Siguiendo la indicación de Eggelhöfer \& Keller Tschirren (50), hemos decidido utilizar la palabra configuración en detrimento de la noción de formación, pese a que esta última es la denominación
} 
traspasar los límites de la discusión en torno a la problemática relación entre la naturaleza y la obra pictórica, haciendo de la organización no sólo un problema artístico y estético, sino también epistemológico y político.

1.

Durante el otoño de 1923, Paul Klee, por entonces profesor titular de la Bauhaus de Weimar, reprochaba a sus estudiantes la falta de vivacidad de sus ejercicios prácticos. El surgimiento del sonido, la germinación y el crecimiento de las plantas, el movimiento de las olas, el curso de los ríos, la circulación sanguínea y la constitución física del cuerpo humano fueron los ejemplos en virtud de los cuales el maestro suizo buscó transmitir a sus estudiantes fenómenos concretos de crecimiento natural (Eggelhöfer \& Keller Tschirren 52). La exhortación resulta entonces clara: "El diálogo con la naturaleza sigue siendo para el artista una condición sine qua non" (Klee 1976 67). Sin embargo, ¿en qué estriba esta apelación a una íntima relación entre el artista y la naturaleza? Pues bien, para Klee es solo a partir de la investigación exhaustiva de la naturaleza que el artista consigue producir configuraciones vivas.

En efecto, si la naturaleza resulta para el artista la mejor escuela de creación, es a partir de su capacidad para producir configuraciones vivas. Ahora bien, esta potencia generativa concedida a la naturaleza pone de manifiesto un tipo particular de concepción de la misma. Para Klee (1976 48), los asuntos relativos a la creación de configuraciones vivas se relacionan, según la expresión spinoziana, menos con una consideración de la naturaleza en tanto "naturalizada" y más con una naturaleza "naturalizante" (Spinoza I, 29). En otras palabras, se trata del rechazo de la naturaleza clausurada y de las organizaciones terminales, en provecho de la apertura al movimiento como eje central en la configuración de formas vivas: la naturaleza en tanto moviente y dinámica. En palabras del propio Klee: "Buena es, por tanto, la forma como movimiento, como hacer; buena es la forma en acción. Mala es la forma como inercia cerrada, como detención terminal [...] La forma es fin, la muerte, la formación [configuración] es vida" (1976 91).

Desde esta perspectiva, Klee parece rechazar aquellas consideraciones que hacen de la naturaleza un dominio armónico de formas dadas, es decir, una naturaleza pensada como una totalidad animada y una unidad en donde nada

utilizada en la versión del texto de Klee utilizada en este escrito. Esta decisión se fundamenta, tal y como indican las autoras, en que la palabra configuración pone el acento en las condiciones previas y los caminos que conducen a la forma, vale decir, acentúa el carácter vivo de la obra pictórica, cuestión que a nuestro juicio se condice con el propio espíritu del artista suizo. De todos modos, hemos decidido preservar en las citas textuales del texto de Klee la palabra formación, añadiendo en corchetes [] la palabra configuración. 
carece de fin. Relevar el movimiento y el devenir de las formas representa aquí una estrategia tendiente a conjurar toda consideración y reducción de la naturaleza al dominio de la teleología. "Nunca, en ninguna parte, la forma es resultado adquirido, acabamiento, remate, fin, conclusión. Hay que considerarla como génesis, como movimiento. Su ser es el devenir [...]” (1976 91). Entonces, si hay algo que el artista aprende en su estudio de la naturaleza es precisamente su carácter moviente, abierto y de duración continuada: la creación como génesis, eje central del trabajo generativo de la naturaleza y, por tanto, del acto de creación en el arte.

Ahora bien, ¿de dónde surge esta verdadera pasión por parte del artista en hacer de la génesis un único y mismo proceso tanto en el quehacer natural como plástico? A contrapelo de la presunta influencia que habrían ejercido sobre Klee los discursos biocéntricos de comienzos del siglo XX (cf. Eggelhöfer 173), esta pretensión parece sostenerse en base a las enfáticas críticas del artista hacia aquel tipo de arte ligado al empobrecimiento formalista y a la representación realista de las cosas. En sus palabras:

Por sobre todo, no hay que dejarse inducir en error y considerar orgánica, por ejemplo, la representación de un cuerpo, con el pretexto de que se puede comprobar la justeza de las proporciones de los dedos en relación a la mano, la de la mano con relación al brazo y al antebrazo, etc. En este caso, se trata del arte de un Ajeno, cuyas obras -simples ejemploseducan para hacer otro tanto, analógicamente con las nociones plásticas (Klee 1976 56).

Ahora bien, ¿Cómo entender esta impugnación de ajenidad establecida por Klee a la representación del cuerpo basada en la organización de sus partes, la proporcionalidad y la unidad? La respuesta a esta pregunta pasa por el estatuto profundamente relativo, vale decir, múltiple, que el artista otorga a lo visible. En efecto, las cosas - y en este caso, el cuerpo- ponen al descubierto una dimensión por entero amplia y compleja, aquella del movimiento como fundamento del devenir (Klee 1976 58), dimensión capaz de invalidar las concepciones racionalistas. En otras palabras, lo orgánico demanda ser entendido aquí en términos de lo viviente, es decir, ligado a la centralidad del movimiento, no solo en la creación artística, sino también en la generación de las cosas, cuestión que, para Klee, resulta especialmente ejemplar en el caso de la configuración pictórica del cuerpo.

Entonces, si la organización y la representación del cuerpo implican una reducción de lo viviente al primado de un cierto telos relativo a la organización, la unidad y la proporcionalidad de sus partes respecto de un todo, la auténtica configuración en movimiento vivo debe tomar los elementos de un organismo sus órganos - y reorganizarlos entre sí (Eggelhöfer \& Keller Tschirren 65). Sin embargo, vale la pena enunciar aquí la siguiente salvedad: esta reorganización del 
organismo, en modo alguno supone un prosaico reemplazo de una organización por otra. Por el contrario, ésta alude a una apertura a la multiplicidad e infinita variabilidad de las cosas, elevando lo accidental al nivel de la esencia (Id. 61).

Así, la configuración pictórica parece presentar cierta cercanía con la noción de cuerpo sin órganos propuesta por Deleuze y Guattari (1985 18-23, 2002 155-172), pues esta última afirmaría, en su estatuto reorganizativo, la irreductibilidad de la multiplicidad, es decir, la primacía de una disparidad de los órganos con respecto a todo principio de organización y de unidad. En consecuencia, siguiendo la expresión propuesta por el filósofo suizo Serge Margel, de lo que aquí se trata es de una remisión infinita mediante la cual los cuerpos (o los órganos) se enmarañarían los unos en los otros, sin que ninguno de ellos se reduzca a la identidad de un solo cuerpo finito, completo, acabado (19).

\section{2.}

El acto creativo en el arte se desplaza, entonces, desde las denominadas formasfines - la organización como clausura y acabamiento- hacia una consideración procesual y abierta, es decir, la configuración por sobre la forma. Según los términos del pintor, "la marcha hacia la forma por sobre el fin terminal, por sobre el final del trayecto, la formación [configuración] determina la forma y es, en consecuencia, predominante" (Klee 1976 91). Ahora bien, marcha, trayecto y recorrido no aluden solamente al proceso de configuración pictórica, por el contrario, suponen remontarse nuevamente a la naturaleza, al proceso de configuración de lo vivo. Tal como la forma de la hoja surge a partir de un impulso dinámico que hace brotar corrientes de energía por sus nervios, emergiendo el tallo a partir del cual se despliegan las hojas y las flores (Eggelhöfer \& Keller Tschirren 52), para Klee, la obra plástica surge a partir de la emergencia de un cierto "fuego que se transmite a la mano, se carga sobre la hoja, desparramándose, ardiendo en chispas" (1976 60).

Atender a esta analogía supone otorgar centralidad a un asunto especialmente relevante para Klee, a saber: la dimensión de las fuerzas, sus recorridos y sus trayectos. Se trata de una ampliación de la comprensión y apreciación de la apariencia desde una mirada centrada en la organización acabada de sus partes hacia la visibilización de las fuerzas que lo configuran como un todo viviente. En sus recorridos y trayectos, las fuerzas y las energías componen una dimensión por entero diferente. Hablamos de una perspectiva 'interna' del objeto, capaz de exceder y subvertir la mera exterioridad de su apariencia. En otras palabras, esta dimensión parece revelar la "estructura o la función material de la cosa” (Klee 1976 69). Tal y como indica Klee: “El mundo no se muestra a los que se distancian de él, para contemplarlo desde una posición externa, como algo 
objetivo; sino a aquellos que ven desde adentro, con sus perspectivas infinitas y sus caminos divergentes" (Id.). De ahí que para el artista suizo el proceso creativo sea menos un asunto de anatomía - en tanto disciplina de la forma, de la estructura y de la organización - y más una cuestión de fisiología y dinámica, es decir, de un interés por las funciones y el funcionamiento mecánico y bioquímico de la materia viva. Efectivamente, un estudio fisiológico dentro del campo del arte concierne a la visualización de las corrientes subterráneas de funciones vivientes, vale decir, a la manifestación de las fuerzas y energías vitales en sus recorridos y trayectos. Un arte plástico dinámico, interno y fisiológico tiene por objeto justamente esa magia inscrita en la materialidad de los objetos, a saber, los trazos de las fuerzas creadoras, sus funciones (Klee 1976 88). En consecuencia, el acto de creación no es otra cosa sino la reactualización y el ingreso de las cosas en la existencia, ahora, en un plano dimensional diferente, aquel de la obra. Pues si las cosas conservan la huella de su movimiento generativo, la tarea del artista es entonces, revivir dicho devenir.

Desde esta perspectiva, el arte plástico implica una ampliación de la apariencia mediante la visibilización de sus funciones. Efectivamente, las fuerzas creadoras son, para Klee, materia en sí. Sin embargo, se trata de un tipo de materialidad no perceptible - a diferencia de lo que ocurre con los otros tipos de materia-, pero que resultaría reconocible en virtud de su encarnación en la materia conocida (Klee 1976 85). Dicho de otro modo, es unida a la materia que las fuerzas creadoras tomarían cuerpo, convirtiéndose en formas, "en realidad". (Id. 88). En tales condiciones, podemos retomar ahora, bajo renovados tintes, la célebre expresión enunciada por Klee y tantas veces retomada por Deleuze (2006 69; 2016 35): “El arte no reproduce lo visible, hace visible" (1976 55). Como se verá más adelante, esta reactualización y visibilización de los trayectos y las fuerzas no solo permite un acercamiento entre Klee y el pintor irlandés Francis Bacon, a quien Deleuze atribuye precisamente el haber hecho visibles las fuerzas, sino también, apostar por una concepción cartográfica de la obra pictórica (1996 93).

\section{3.}

En Mil Mesetas Gilles Deleuze y Félix Guattari introducen su noción de ritornelo a través de la experiencia que tiene un niño que teme a la oscuridad y el modo en que la repetición de un canturreo le permite calmarse organizando su entorno, hasta ese momento caótico.

Un niño en la oscuridad, presa del miedo, se tranquiliza canturreando. Camina y se detiene de acuerdo con su canción. Perdido, se cobija como puede o se orienta a duras penas con su pequeña canción. Ésta es como el esbozo de un centro estable y tranquilo, estabilizante y 
tranquilizante, en el seno del caos. Puede ser que el niño salte al mismo tiempo que cante, acelere o aminore su paso; pero la canción ya es en sí misma un salto: salta del caos a un comienzo de orden en el caos, pero también corre el riesgo de dislocarse a cada instante (2002 382).

Aquí, el caos aparece como un inmenso agujero negro respecto del cual nos esforzaríamos por fijar un punto como centro, aun cuando este acto sea siempre un intento del todo frágil y precario. Al respecto, es interesante que el propio Deleuze (2006 38) atribuya a Paul Klee el haber destacado que el caos sería algo así como el "centro de una balanza" (Klee 1976 84). Acto seguido, el filósofo hace aparecer con toda su fuerza las palabras del pintor suizo:

Establecer un punto en el caos es reconocerlo necesariamente gris en razón de su concentración principal y conferirle el carácter de un centro original desde donde el orden del universo va a brotar e irradiar en todas las dimensiones. Afectar un punto de una virtud central es hacer de él el lugar de la cosmogénesis. A este advenimiento corresponde la idea de todo Comienzo (Id. 84).

Sin embargo, lejos de concernir a una pura negatividad y a una especie de anodina ausencia, el caos es, desde la perspectiva del artista, un punto gris. Punto gris, pues emerge como un nodo en donde se intersectan heterogéneas dimensiones pictoricas configuradas como series de opuestos: blanco y negro, arriba y abajo, cálido y frío. Siguiendo a Klee:

Es gris pues no es blanco ni negro, o porque es tan blanco como negro. Es gris porque no está arriba ni abajo, o porque está tan arriba como abajo. Gris porque no es cálido ni frío. Gris porque es punto no dimensional, punto entre las dimensiones y en sus intersecciones, o en el cruce de los caminos" (Id. 84).

Al igual que el niño en la oscuridad descrito por Deleuze y Guattari, para Klee el punto gris salta "por encima de sí mismo en la dimensión donde crea el orden" (1961 60). Por un lado, el salto del niño en su canción, por el otro, el salto del punto por encima de sí mismo: lógica diagramática (Deleuze 2006 43) en virtud de la cual, a partir del caos, emerge 'otra cosa'; el orden, la obra pictórica.

$\mathrm{Al}$ respecto, resulta relevante constatar cómo Klee hace del punto gris, en tanto punto de génesis, de creación en movimiento, sinónimo del huevo [óvulo] (1976 85). Ello, pues el ya mencionado cuerpo sin órganos es descrito por Deleuze y Guattari bajo los mismos términos, a saber, como el huevo (2002 158, 1985 27). Cabe entonces la posibilidad de pensar este punto de caos-génesis descrito por el artista en su correspondencia con aquello que los filósofos franceses describieron como cuerpo sin órganos, ese punto paradójico que, si bien es por naturaleza improductivo, emerge como el punto de inicio de toda producción: "El cuerpo sin 
órganos es un huevo: está atravesado por ejes y umbrales, latitudes, longitudes, geodésicas, está atravesado por gradientes que señalan los devenires y los cambios del que en él se desarrolla" (Id. 27). Así, es a partir de la sugestiva noción de huevo que, tanto el cuerpo sin órganos como el punto gris, aparecen como el genuino 'inicio' de todo devenir. Punto gris y cuerpo sin órganos, definirían entonces un lugar de encuentro entre el filósofo y el artista: punto de partida de la creación desde del caos.

Este espacio de encuentro entre los dos pensadores de la creación pone de manifiesto, en primer lugar, una crítica a quienes toman lo ya organizado como punto de partida para entender la generación de los cuerpos o las figuras $y$, en segundo lugar, una nueva concepción de lo vital, la cual se corresponde con lo que Deleuze denomina -inspirado en el teórico del arte Wilhelhm Worringer - "vida no-orgánica" (Deleuze Francis Bacon 29, Deleuze \& Guattari Mil Mesetas 412). En efecto, la vida no-orgánica puede ser pensada como aquello que define el carácter vital que descubren tanto Worringer como Klee y Deleuze en la creación artística, pues en cada uno de estos autores se trata de pensar la vida más allá de su organicidad y unidad. Bajo tales condiciones, la figura del huevo emerge como metáfora de una potencia todavía no orgánica en virtud de la cual empieza una vida.

Para Klee, el huevo es el la imagen de lo que, si bien no excluye el caos, es también punto de cosmogénesis. Aún más, en su carácter a-dimensional, el punto gris es aquel desde donde surgen todas las dimensiones: punto inmóvil a partir del cual se inicia todo movimiento (1976 84). En su estatuto a la vez genético y caótico, el punto gris podría ser entendido como aquello que Deleuze y Guattari designaron con el neologismo "Caosmos", aquello que tienen en común el caos y el ritmo, el entre-dos, el entre dos medios (Deleuze \& Guattari 2002 320). Asimismo, en el punto gris, cuerpo sin órganos o huevo se da una distribución de diferencias de manera tal que ninguna cualidad fija, ningún tiempo medido se remiten a un objeto identificable o reconocible: en el huevo se confunden las partes, resultando desestimada toda posibilidad para la producción de una organización preestablecida o la emergencia de un conjunto [unidad] compuesto de órganos diferenciados. En su potencia, tanto el punto gris como el cuerpo sin órganos, nos situarían entonces, ante la posibilidad de una configuración hecha únicamente de "singularidades móviles" (Deleuze 1994 97).

Huevo y punto gris son entonces, un centro intenso y vivo de la materia que define la dimensión de una vida no-orgánica. Y es que, lo orgánico se vincula, tanto en Klee como en Deleuze, con las formas fijas, meros resultados de un proceso de génesis. No por nada, a fin de graficar dicha distinción, Deleuze y Guattari (2002 373) opondrán al círculo el redondel. Mientras el primero no es más que esencia fija y orgánica, el segundo reafirma su esencia vaga y fluyente, 
distinguiéndose radicalmente tanto del círculo como de todo el resto de figuras redondas (un vaso, una rueda, el sol, etc.). Por su parte, Klee (1961 60) opone al formalismo de las formas exactas -aquellas que es posible encontrar (quiéraselo o no) en los objetos que percibimos-las formas vivas, siempre dotadas de una función. Así, se entiende entonces la conjura de Klee a todo formalismo, pues "el formalismo es la forma sin la función" (82). En última instancia, de lo que se trata es de "líneas que ya no limitan ninguna forma, que ya no forman ningún contorno, colores que ya no distribuyen paisaje (es la semiótica pictórica)" (Deleuze \& Guattari 2002 300). Es en este sentido que el arte no es ni figurativo ni orgánico, siguiendo las palabras del propio Klee:

Los elementos específicos del arte gráfico son puntos y energías lineales, planas y espaciales. Ejemplo de elementos planos que no se deja descomponer en unidades subordinadas: la energía, uniforme o modulada, surgida de una punta ancha (1976 56).

Podríamos pensar que es esto lo que Deleuze ve tanto en Paul Klee como en Francis Bacon: la presentación de un cuerpo "que intenta escapar por uno de sus órganos, para reunirse con el fondo liso, la estructura material" (2016 24). En efecto, el trabajo plástico propuesto por el artista suizo demanda ser entendido como la experiencia de un cuerpo que trata de escapar a lo figurativo, a las formas estáticas y a cualquier principio tendiente a la organización; mostrando que el arte - tal y como señalamos anteriormente-, no busca reproducir lo visible, sino que, "hacer visible" (Klee 1976 55). Es desde esta perspectiva que el trabajo pictórico sería menos un asunto de transformación que de deformación. Ello, pues de lo que se trataría es de llevar a un cuerpo a su punto límite, a su punto de noorganización, generando a partir de ahí nuevas configuraciones. En otras palabras, se trataría de captar el carácter de multiplicidad que todo cuerpo lleva consigo, a saber, reactualizar y hacer visibles esas fuerzas y trayectos profundamente materiales que se encontrarían encarnados en la materia conocida.

\section{4.}

Es a partir del carácter múltiple concedido a todas las cosas que resulta posible una comprensión propiamente moderna del arte, ya sea de las denominadas 'artes espaciales' como de la música, 'arte del tiempo', pues ambas consisten en la concomitancia de múltiples dimensiones en un mismo complejo; cuestión que la música alcanza en virtud de la polifonía y la pintura a partir de la denominada “polifonía plástica” (Klee 1976 63). De acuerdo a lo anterior, cabe indicar entonces, que tanto en Klee como en Bacon el proceso creativo introduciría, inmanentemente, "una deformación, una convulsión, una explosión, en una 
palabra, un movimiento de extrema violencia" (Deleuze \& Guattari 1985 258). De hecho, el artista suizo fue especialmente enfático en este punto, toda vez que el acto de creación, a saber, el paso de las cosas por la discontinidad dimensional entre el orden natural y el orden plástico, es un acontecimiento radicalmente deformante. En tal sentido, la auténtica configuración pictórica no es otra cosa sino la "resurrección de la naturaleza" (Klee Teoría 38) por la vía de su deformación.

En definitiva, la creación artística parece poner en obra algo que está presente en todo proceso de producción a distintos niveles de lo existente. Se trata, de un devenir que pone en marcha los componentes materiales de la naturaleza, los cuales hayan su expresión en la obra artística, pero también en cualquier forma percibida. Es esto lo que define - a juicio de Klee (1976 25) - al expresionismo como invocación de un punto de génesis en la obra pictórica. Vale decir, es en este preciso sentido, y no en un sentido científico, que el artista se interesa entonces en la naturaleza.

Siguiendo esta serie de proposiciones, cabría retomar aquí una especial consideración realizada por Deleuze (2016 23) respecto a la obra de Francis Bacon, se trata del desplazamiento desde el problema del lugar del cuerpo al problema del acontecimiento. De acuerdo a lo anterior, podríamos atribuir al arte no sólo una humilde tarea de visibilización, sino que, la particular labor de hacer visible el acontecimiento, es decir, aquello que ocurre en una "superficie invisible, incorporal" (Deleuze 1994 226). Hacer visible, hacer visible el acontecimiento, esa sería la difícil tarea que es posible atribuir al proyecto creativo emprendido por Klee: "Arte o naturaleza, es difícil abarcar con la mirada un conjunto de este tipo, y más difícil aún es facilitar a otro su visión" (1976 40).

Hacer visible sería, entonces, encarnar el acontecimiento, actualizar lo virtual, visibilizar las fuerzas que, en palabras de Klee, atraviesan los objetos. Así también, hacer visible aquellas fuerzas que, a juicio de Deleuze, atraviesan las partes del cuerpo, los órganos que se determinan en función de elementos deformadores que los afectan: "una cabeza a la que le son agregados óvalos y trazos para desorbitar los ojos, inflar los orificios de la nariz, prolongar la boca, movilizar la piel, en un ejercicio común de todos los órganos a la vez" (Deleuze 2016 46). Ciertamente, esto no quiere decir que no haya una lógica involucrada en el trabajo que lleva a cabo el artista con los materiales, es decir, en esa transposición de dimensiones y formas que va desde la naturaleza a la obra pictórica (Klee 1976 36). De hecho, es a partir de Deleuze que dicho quehacer cabría ser pensado como una particular lógica de la sensación $\mathrm{o}$, incluso, una lógica propiamente diagramática. Con esto, buscamos aludir a la dimensión del orden devenido del caos, al salto del punto gris sobre sí mismo (Klee 1961 60) es decir, a la distribución en el cuadro de las intervenciones que, de otra manera, serían puramente azarosas; cuestión que precisamente posibilita su emergencia en tanto 
que hechos pictóricos (Deleuze 2006 43). En efecto, es a partir de esta lógica diagramática que el artista logra sustraer el cliché, condición de posibilidad del advenimiento de lo nuevo, lo inédito, la visibilización de algo en el orden del acontecimiento.

Bajo tales condiciones, podemos retomar entonces no solo la crítica al arte representacional propuesta por Klee, sino también resaltar el estatuto profundamente no-representacional de su propio trabajo pictórico. En efecto, esta naturaleza no-representacional de la obra de Klee contiene - a nuestro juicio - una profunda presencia en la comprensión deleuzeana de lo virtual en su relación a la multiplicidad y al acontecimiento. $Y$ es que, en la medida que lo virtual es lo que no se realiza totalmente, es por lo mismo a-representacional. Lo virtual no puede ser representado, lo virtual solo puede ser presentado. Por consiguiente, la pintura, en la medida en que hace eco de un acontecimiento virtual, no sigue una lógica representacional. De ahí, la apelación del filósofo a un arte impersonal y cartográfico, conjuración radical a las concepciones conmemorativas, representacional y arqueológicas dentro del quehacer artístico.

\section{Conclusiones}

¿Cuándo ocurre el acto creativo en pintura? Para Deleuze, el acontecimiento pictórico implica siempre una catástrofe. Se trata del paso por el caos que permite deshacerse de lo que precede, de todo aquello que pesa sobre el cuadro aún antes de que éste haya comenzado (2006 42). Desplome de los datos figurativos y de la estructura narrativa en tanto que, proceso preparativo - y normativo, por ciertodel acto pictórico. Esta operación de cosmogénesis que Deleuze no sólo extrae de Klee sino también de Cézanne y Bacon nos ha interesado por su carácter eminentemente análogico (Deleuze 2006 127, 2016 115). Tal y como el grito de Artaud, lo analógico acaece como una presentificación inmediata, es decir, como la producción de una semejanza que, sin embargo, no se encuentra mediatizada por un código y ni precedida por unidades convencionales. En su carácter analógico, el acto creativo es entonces, la producción de una semejanza sin común medida, sin molde ni ejemplo.

De esto se trata, precisamente, la conjura que -a nuestro juicio- atraviesa buena parte del proyecto pictórico y teórico de Klee. Para el artista suizo el arte no educa, en tanto apuesta disciplinar tendiente a la reducción del arte a la reproducción de un modelo. Justeza, proporcionalidad entre sus partes y unidad - en suma, organización-, son aquí un acabamiento de la vida, reducción de la vitalidad de la configuración a la reproductibilidad de las denominadas formasfines. Desde esta perspectiva, la crítica radical a la organización propuesta por Klee y relevada por Deleuze permite no sólo otorgar centralidad a la diferencia, la 
multiplicidad y el devenir dentro del campo del arte, interrogando con ello el fundamento representacional del arte pictórico, sino también, situar la obra de Klee como un apuntalamiento de la "inversión del platonismo" promulgada por Deleuze (1994 186). En efecto, tal y como el simulacro deleuzeano (Id. 186, 2017 197), las auténticas configuraciones vivas propuestas por Klee emergen bajo la égida de una disimilitud y discontinuidad interior radical respecto de la naturaleza. En su carácter polifónico, relativo y de deformación, la configuración pictórica no compone las partes bajo la primacía de una unidad, afirmando así su derecho por sobre los ejemplos, las copias y los íconos.

Es a partir del carácter de deformación de toda configuración pictórica que entendemos el comentario de Michel Foucault respecto a la obra de Klee. Esto, pues para el filósofo francés, el gesto pictórico de Klee supone una subversión de la jerarquía y la distinción entre el discurso y la forma mediante la configuración de una polifonía en donde se yuxtaponen "las figuras y la sintáxis de los signos" (29). Deformación de los cuerpos como índice de un devenir. Devenir corpóreo, encarnación o actualización de un acontecimiento virtual. Es decir, aquello que Klee presenta como "la brecha abierta por esa fuerza inversa que humaniza las cosas divinas al deformarlas para manifestárnoslas" (1976 100). He ahí la apuesta tendiente a conjurar la legitimidad de 'nuestra escala' como medida de todas las cosas: la organización y la representación como clausura, meta y finalidad. Si toda configuración es una deformación, se trata, pues, de la efectuación del acontecimiento o de la actualización de lo virtual a partir de un proceso de producción que hace surgir las formas inscritas en los cuerpos. Proceso que se hace evidente en el acto creativo en el arte pictórico a partir de la actualización y visibilización de las fuerzas y sus trayectos en la materialidad de la obra.

Por consiguiente, lo que habrían hecho tanto Deleuze como Klee sería un retorno a las fuentes de la creación, ahora, bajo la premisa de la diferencia, el movimiento y la multiplicidad (Lapoujade 12, Sauvagnargues 11). Proceso que va contra la organización en la medida que consiste en hacer escapar a un cuerpo a través de uno de sus órganos, es decir, someter a transformación las formas fijas involucradas en la organización de un cuerpo. Así, toda producción implica pasar por un punto intensivo, no-organizado, de la materia. Cuerpo sin órganos en Deleuze (1985 18-23, 2002 155-172), punto gris en Klee (1976 84), y que en ambos es la noción de huevo. Centro germinal de distribución de diferencias que no aguanta ninguna cualidad fija. El caos como génesis del cosmos. El arte como la instauración de un centro de ampliación de la materia. Arte que funciona como lo vivo en su dinamismo, es decir, en el sentido de que la vida es un proceso siempre abierto en el que la configuración es siempre una deformación. 


\section{Bibliografía}

Artaud, Antonin. "Un pintor mental". Teoría del arte moderno. Buenos Aires: Caldén, 1976. 23-24.

Deleuze, Gilles. Lógica del sentido. Barcelona: Paidós, 1994

Deleuze, Gilles. Crítica y clínica. Barcelona: Anagrama, 1996.

Deleuze, Gilles. Pintura. El concepto de diagrama. Buenos Aires: Cactus, 2006.

Deleuze, Gilles. Francis Bacon. Lógica de la sensación. Madrid: Arena Libros, 2016.

Deleuze, Gilles. Diferencia y repetición. Buenos Aires: Amorrortu, 2017.

Deleuze, Gilles \& Guattari, Félix. El Anti-Edipo. Capitalismo y Esquizofrenia. Barcelona: Paidós, 1985.

Deleuze, Gilles \& Guattari, Félix. Mil Mesetas. Capitalismo y Esquizofrenia. Valencia: Pre-Textos, 2002.

Eggelhöfer, Fabienne \& Keller Tschirren, Marianne. “Teoría de la configuración pictórica. Paul Klee. Maestro de la Bauhaus, ed. Fundación Juan March. Madrid: Fundación Juan March, 2013. 49-126.

Foucault, Michel. Esto no es una pipa. Ensayo sobre Magritte. Buenos Aires: Eterna Cadencia, 2012.

Klee, Paul. Notebooks Volume 1. The thinking eye. Londres: Lund Humphries, 1961.

Klee, Paul. Teoría del arte moderno. Buenos Aires: Caldén, 1976.

Lapoujade, David. Deleuze. Los movimientos aberrantes. Buenos Aires: Cactus, 2016

Margel, Serge. Alienación. Antonin Artaud. Las genealogías híbridas. Santiago: Metales Pesados, 2016.

Sauvagnargues, Anne. Deleuze. Del animal al arte. Buenos Aires: Amorrortu, 2006.

Spinoza, Baruch. Ética demostrada según el orden geométrico. Madrid: Editora Nacional, 1980. 